\title{
Status Epilepticus and Coma in Pregnancy. Management Dilemma in a Resource Limited Setting (Monatele, Cameroon): Case Report
}

\author{
Mosman Anyimbi Ofeh ${ }^{1,2^{*}}{ }^{(}$, Charles Regent Kenne Nodem² \\ ${ }^{1}$ Department of Obstetrics and Gynecology, Higher Institute of Medical Technology of Nkolondom, Yaoundé, Cameroon \\ ${ }^{2}$ Internal Medicine, Gynecology and Obstetrics Unit, Monatele District Hospital, Monatele, Cameroon \\ Email: *mosmanofeh@yahoo.com
}

How to cite this paper: Ofeh, M.A. and Nodem, C.R.K. (2022) Status Epilepticus and Coma in Pregnancy. Management Dilemma in a Resource Limited Setting (Monatele, Cameroon): Case Report. Open Journal of Obstetrics and Gynecology, 12, 25-32. https://doi.org/10.4236/ojog.2022.121003

Received: November 3, 2021

Accepted: January 10, 2022

Published: January 13, 2022

Copyright $\odot 2022$ by author(s) and Scientific Research Publishing Inc. This work is licensed under the Creative Commons Attribution International License (CC BY 4.0).

http://creativecommons.org/licenses/by/4.0/

\begin{abstract}
Epilepsy is a leading neurological condition characterized by recurrent seizures and affecting more than 50 million people worldwide. Status epilepticus (SE) is a neurological emergency associated with a high mortality rate and long-term cognitive sequelae. In pregnancy, status epilepticus poses a tremendous threat to both mother and fetus. We report a case of status epilepticus in pregnancy complicated by coma, where obstetrical ultrasound revealed fetal demise in utero followed by rapid maternal deterioration and demise later. There was management challenge of a comatose pregnant mother in very poor and deteriorating hemodynamic state with fetal demise in a low economic and limited resource setting.
\end{abstract}

\section{Keywords}

Status Epilepticus, Pregnancy, Coma, Fetal Demise, Epilepsy

\section{Introduction}

Epilepsy is a leading neurological condition which is characterized by recurrent seizures and affecting more than 50 million people worldwide. There is an important geographical burden of epilepsy, with most of the cases (80\%) living in low- and middle-income countries [1]. Status epilepticus (SE) is a neurological medical emergency associated with a high mortality rate and long-term cognitive sequelae. The definition of SE has undergone several modifications which include mainly the duration of seizures, the type of seizure, the clinical state of the patients, the time necessary to intervention and response to treatment. The International League Against Epilepsy (ILAE) defines SE as a condition resulting 
either from the failure of the mechanisms responsible for seizure self limitation or from the initiation of mechanisms which lead to atypically prolonged seizures [2] [3]. Here, we defined SE as a continuous seizure lasting more than $30 \mathrm{~min}$, or two or more seizures without full recovery of consciousness between any of them [3]. The seizures of SE can be either focal or generalized in onset and either convulsive or nonconvulsive in presentation. During SE, consciousness can be preserved (e.g., focal motor SE), altered (e.g., complex partial SE), or completely lost in the absence of motor symptoms (e.g., absence SE or electrographic SE in the comatose patient [2].

The causes of SE vary with age. Common identifiable causes of SE include trauma, tumors, vascular disease, alcohol withdrawal, infections and noncompliance with antiseizure medications. Status epilepticus occurs at all ages, but is most common at the extremes of life [2]. Pregnancy-related SE is a rare condition that has been reported to occur in $0.6 \%$ of all patients with epilepsy during pregnancy [4]. It poses a tremendous threat to both mother and fetus [4] as pregnancy is associated with a number of physiological, endocrine and psychological changes, any or all of which might contribute to lowering the seizure threshold with resultant frequent seizures [5]. A high epilepsy prevalence varying from $4.6 \%$ to $7.8 \%$ has been reported in several onchocerciasis-endemic locations along the Sanaga river in Cameroon [6]. Monatélé, capital of the Lekie division of the center region of Cameroon is one of these towns along the river Sanaga.

We present a case of status epilepticus and coma complicated by intrauterine fetal demise in pregnancy in a resource limited setting in Monatélé, Cameroon. The aim of this presentation is to show possible fatal complications and management dilemma of pregnant epileptic women in a low resource setting.

\section{Case Presentation}

Miss N.M. 27 years old G7 P6004. She was brought to the Monatélé District hospital by her uncle and aunt for five days of seizures and unconsciousness. Her care takers reported seizures which began five days prior to consultation, estimating the total number of seizures to about thirty and describing tonic clonic seizures lasting about 3 to 5 minutes with post ictal unconsciousness. They also reported that she had fallen from her bed during some of these episodes.

She was a known epileptic, diagnosed at the age of 11, irregularly followed up, on two antiepileptic drugs: Carbamazepine $200 \mathrm{mg}$ tablet BID (twice daily) and Phenobarbital $100 \mathrm{mg}$ tablets BID. This treatment had been interrupted a few days before seizures due to financial difficulties. Her last reported seizure before these episodes was more than three months ago.

She was G7P6004 with an unknown last menstrual period and gestational age, but family members estimated the pregnancy to about seven months. She had six live births all by normal vaginal delivery and lost two children as toddlers due to illness. The other four were in good health apparently. Her pregnancy was not 
being followed up as she had not attended any antenatal clinic. She had no surgical history. It was not certain whether she used a contraceptive method before pregnancy, whether she used treated mosquito bed net or not, whether she had an allergy and whether she consumed alcohol and/or tobacco during pregnancy or not. There was no medical consultation book available. She was single and lived with her mother.

On entry physical examination revealed an unconscious woman with a Glasgow Coma Scale $(\mathrm{GCS})=9 / 15$ (eye opening $=4$, verbal response $=2$, movement $=$ 3). A temperature of $40.4^{\circ} \mathrm{C}$, blood pressure $105 / 67 \mathrm{mmHg}$, pulse rate $146 \mathrm{bpm}$, respiratory rate $28 \mathrm{~cm}$ and $\mathrm{SpO}_{2}$ at $74 \%$. Her weight was not measured. Her conjunctivae were red and there was no scleral jaundice. There was a whitish-yellowish discharge on the sclera. There was bilateral non-reactive miosis and some dry blood stains on the left angle of her lips. Thorax and breast examination had no significant findings apart from superficial respiration. Her abdomen was distended by a gravid uterus with a fundal height of $27 \mathrm{~cm}$ and an estimated fetal weight of 2187 grams. There was no fetal heartbeat heard on auscultation and no fetal quickening perceived. The vulva was clean with no stains. Digital vaginal examination revealed a posterior closed and long cervix.

Diagnosis made were Status epilepticus complicated by Stage 1 coma and suspected intrauterine fetal demise with etiology of status epilepticus being bacterial meningitis, severe malaria, intracranial hemorrhage from the several falls or antiepileptic drug withdrawal. Workup consisted of Malaria thick blood film, obstetrical ultrasound, random blood sugar, Human Immunodeficiency Virus serology and Hemoglobin level (Table 1). Computer tomography and analysis of cerebrospinal fluid are not available in the Hospital. Immediate management consisted of a peripheral intravenous line with $500 \mathrm{ml}$ of lactated ringer solution planned over 24 hours, oxygen therapy with oxygen extractor at $4 \mathrm{~L} / \mathrm{min}$, Phenobarbital $200 \mathrm{mg}$ diluted in $20 \mathrm{ml}$ of $5 \%$ glucose solution with a rate of 1 $\mathrm{mL} / \mathrm{min}$ and $500 \mathrm{mg}$ of IV Metamizol. Oxygen saturation, level of consciousness, blood pressure, pulse rate and respiratory rate were being monitored.

An hour after hospitalization we received the Obstetrical ultrasound which showed a 31 weeks +2 days pregnancy, female fetus with no signs of fetal cardiac activity and fetal quickening confirming intrauterine fetal demise. We then referred the lady to a better health structure with an intensive care unit in Yaoundé but due to financial and transportation difficulties and after family agreement the patient was not transferred. On the second hour of admission we received the malaria thick blood film requested which showed 1 - 2 trophozoites of plasmodium falciparum (TPF) per high power field (HPF) (Figure 1). Fasting blood sugar (FBS) was $0.87 \mathrm{~g} / \mathrm{L}$ and hemoglobin level $10 \mathrm{~g} / \mathrm{dL}$ (Table 1). Glasgow coma scale score was $7 / 15$ (eye opening $=3$, verbal response $=2$ and movement $=2$ ). Oxygen saturation was $74 \%$. Malaria treatment with Artemether protocol was started. She received $160 \mathrm{mg}$ of artemether intramuscularly (protocol chosen to minimize cost). The questions we pondered on at this point were if we 


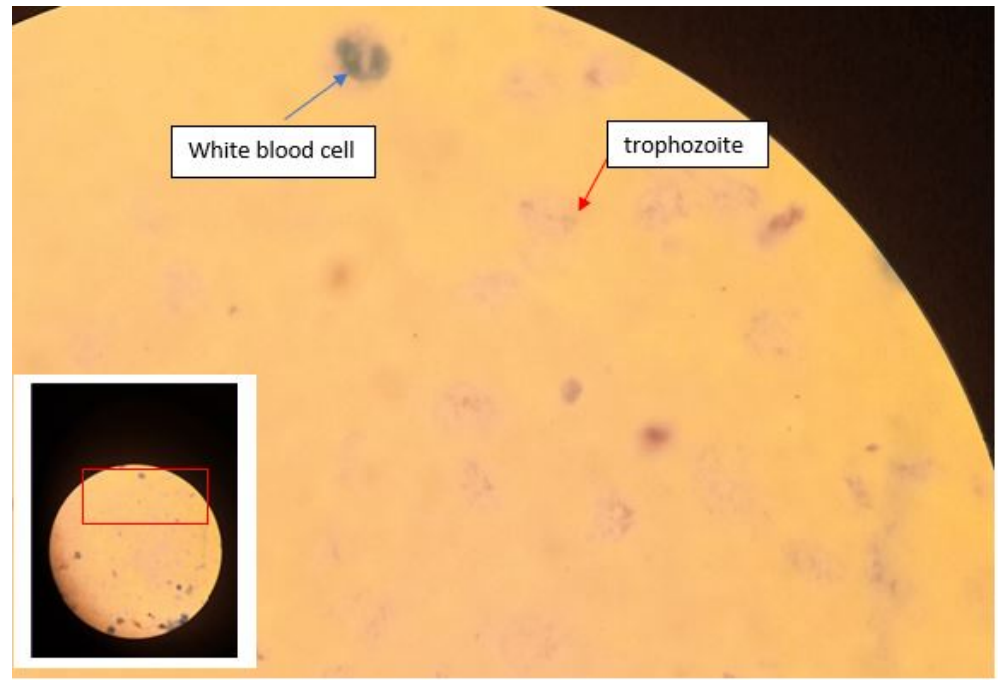

Figure 1. High power field of light microscope at $\times 40$ magnification. Red arrow showing trophozoite of Plasmodium falciparum.

Table 1. Laboratory investigations and results.

\begin{tabular}{lcc}
\hline & Laboratory investigation & results \\
\hline $\mathbf{1}$ & Malaria thick blood film & $1-2 \mathrm{TPF} / \mathrm{HPF}$ \\
$\mathbf{2}$ & FBS & $0.87 \mathrm{~g} / \mathrm{L}$ \\
$\mathbf{3}$ & Hemoglobin level & $10 \mathrm{~g} / \mathrm{dL}$ \\
$\mathbf{4}$ & Human Immunodeficiency Virus 1/2 serology & Negative \\
\hline
\end{tabular}

should induce labor despite her condition, wait and hope for her to regain consciousness before inducing or wait for labor to start naturally. Prothrombin time and cephaline activated time were requested to see if the intrauterine fetal demise had resulted in a coagulopathy that could complicate induction of labor. Unfortunately, the requested tests were not done due to financial difficulties and the absence of the latter in the hospital laboratory.

On the seventh hour of hospitalization a quick reevaluation was done. Glasgow coma scale score then was $5 / 15$ (eye opening $=2$, verbal response $=2$, movement $=1$ ). Respiratory rate was $34 \mathrm{c} / \mathrm{min}$ and temperature 39.5 degrees Celsius. On the eighth hour of admission we were called by her family members for an absence of respiratory movements. Glasgow coma scale score then was 3/15. There was no respiratory movement, no heartbeat and bilateral non-reactive mydriasis. Death was confirmed clinically.

\section{Cause of Death}

The etiology of death in this context could not be determined with certainty. However, several interwoven hypotheses were made: anoxia/brain damage from prolonged seizures, probable cerebral hemorrhage from a head trauma during a fall during the seizures, severe malaria or bacterial meningitis causing seizures 
and coma or respiratory failure from severe brain damage. The context (low financial and technical) did not permit us to confirm or exclude some of these hypotheses.

\section{Discussion}

Epilepsy is defined as repeated (at least 2), spontaneous, unprovoked seizures of any type. Status epilepticus is a neurological emergency which is associated with a high mortality rate ( $9 \%-40 \%)$ and cognitive sequelae [4]. Convulsive SE during pregnancy poses a tremendous threat to both mother and fetus.

Our patient was aged 27 years old falling within the age group with the highest number of epileptic patients in other studies in Cameroon and Taiwan [4] [6] (20 - 29 years and 21 to 36 years respectively). The gestational age after ultrasound was 31 WA (third trimester). A study in Taiwan reported a gestational age ranging from 5 weeks to 26 weeks among 5 pregnant women with status epilepticus while another study reported $17.2 \%$ each in first and third trimesters with up to $51.7 \%$ cases of SE in the postpartum period [4] [7]. Our patient had preexisting epilepsy. Studies in Cameroon and Nigeria have reported patients who had preexisting epilepsy developed status epilepticus [8] [9].

Our patient was on bitherapy and poorly followed up. In other literature monotherapy should always be attempted initially, with multidrug therapy reserved for patients with persistent seizures or unacceptable side effects [10]. The fact that our patient was on a bitherapy (carbamazepine and phenobarbital) surely meant that her seizures could no longer be controlled with monotherapy. Studies on Carbamazepine are mixed in terms of teratogenicity, phenobarbital is effective for partial and generalized tonic-clonic seizures. These Anti-Epileptic Drugs (AEDs) are not ideal during pregnancy but are largely used because of their low cost and they are often the only available AEDs in many developing countries [5] [10].

The diagnosis of Status epilepticus in this case was justified as it is a clinical diagnosis with defined criteria. Given the clinical picture and workup, four etiologies of status epilepticus could be incriminated in this case: either severe malaria, brain trauma, bacterial meningitis, antiepileptic drug withdrawal or a combination of any of these. These etiologies were reported in another study. Anti-seizure medication changes (withdrawal, change) in $15.1 \%$ of cases, brain trauma in $13.2 \%$ of cases, bacterial meningitis in $3.8 \%$ and severe malaria in $1.9 \%$ [9]. Another study reported $10 \%$ of cases related to AED withdrawal [7].

This patient's clinical state of coma with persistent hyperthermia, hypoxemia corresponds to the second phase of SE which is characterized by the failure of cerebral autoregulation, decrease in cerebral blood flow and increase in intracranial pressure. During this phase, electromechanical dissociation may occur in which, although electrical cerebral seizure activity continues, the clinical manifestations may be restricted to minor twitching [3].

The management of our patient included administration of Phenobarbital, 
chosen for its availability. In a study in India the most common AED used for treatment of SE was phenytoin/fosphenytoin followed by phenobarbitone (phenobatital) and levetiracetam [7]. Management of intrauterine demise at the time of discovery was really challenging given the mother's clinical state and the lack of specific workup. A study in Taiwan reported Cesarean section in 2 of 5 patients in preterm because of poorly controlled SE [4]. To our knowledge, there is no clear protocol for management of intrauterine fetal demise in comatose pregnant women especially in a resource limited setting. However, reports have been made of comatose non-epileptic women carrying on with pregnancy to term and giving birth spontaneously [11] [12]. The questions we pondered on were if we should induce labor despite her condition, if we should be expectant for her to regain consciousness before inducing labor, if we should wait for labor to start naturally or if we should go in for a cesarean section. All four options revolved around the fact that the patients clinical state was poor and deteriorating, we lacked specific decisive workup and there was a huge financial difficulty. Our patient also received Artemether which is acceptable for the management of Severe malaria in pregnancy [13].

Our patient was a gravida 7 but still single, concurring to the reality of most epileptic women in Cameroon and Nigeria. This is mostly due to the stigma and beliefs attached to epilepsy [1]. Our patient had not attended any antenatal clinics. This may be due to her relatively low economic status or as a result of stigma related to epilepsy in the community. A study in sub-Saharan Africa reported that women with epilepsy attend less antenatal clinics [1]. There was a huge delay (5 days) in seeking health care from the hospital. This may be due to the low economic status, ignorance of the family or difficulties in transportation as she became unconscious. Epileptic women and their families should be educated on the benefits of following up their pregnancies. There should be a close follow up of epileptic patients by health personnel, especially for those who are pregnant or who plan on getting pregnant.

Although this case ended up in death, not all women with status epilepticus have poor outcomes. One study reports a 10-fold increase risk of death [5] and another reported an overall mortality rate of $28.5 \%$, with $80 \%$ of the survivors returning to baseline [4]. Pregnant patients with SE should be hospitalized in the Intensive Care Unit (ICU). The fact that this patient was not in an ICU greatly reduced her chances of survival as management was not optimal due in part to low technical plateau and economic level. The fetal outcome of this pregnancy was fetal demise days before maternal death. This may have been caused by the poor circulation to the placenta during the seizure episodes. A study in Taiwan reported a series of outcomes from cesarean section, to preterm delivery, delivery at term and to intrauterine fetal death [4]. In another study $76 \%$ of women had good outcome. Majority of the fetuses had good outcomes [7].

A patient in such a clinical picture and the circumstances described is difficult to manage. The points we pondered on were if we should induce labor despite 
her condition, wait and hope for her to regain consciousness before inducing or wait for labor to start naturally.

\section{Conclusion}

Status epilepticus though manageable remains a challenging condition especially in low resource settings. In pregnancy, insufficient, late or poor management can result in perilous outcome for both mother and fetus. Medical societies of Gynecology and Neurology should develop a protocol for the management of fetal demise in comatose women or women with uncontrolled status epilepticus. This will contribute greatly to the third objective of sustainable development.

\section{Authors' Contribution}

Ofeh received, managed the patient and did the write-up. Kenne contributed in the write up.

\section{Acknowledgements}

We would like to thank the patient care takers who despite the difficult situation gave their consent.

\section{Ethical Consideration}

Two weeks later, we obtained verbal consent from one of the deceased uncles who brought her to the hospital.

\section{Conflicts of Interest}

The authors declare no conflicts of interest regarding the publication of this paper.

\section{References}

[1] Menon, S., Siewe, F.J.N., Weckhuysen, S., Bhwanad, D., Njamnshie, A.K., Dekkeret, M., et al. (2019) Women with Epilepsy in Sub-Saharan Africa: A Review of the Reproductive Health Challenges and Perspectives for Management. Seizure, European Journal of Epilepsy, 71, 312-317. https://doi.org/10.1016/j.seizure.2019.08.016

[2] Seinfeld, S., Goodkin, H.P. and Shinnar, S. (2016) Status Epilepticus. Cold Spring Harbor Perspectives in Medicine, 6, a022830. https://doi.org/10.1101/cshperspect.a022830

[3] Ajith, C. and Sanjeev, V.T. (2009) Status Epilepticus. Annals of Indian Academy of Neurology, 12, 140-153. https://doi.org/10.4103/0972-2327.56312

[4] Lu, Y.T., Hsu, C.W., Tsai, W.C., Cheng, M.Y., Shih, F.Y., Fu, T.Y., et al. (2016) Status Epilepticus Associated with Pregnancy: A Cohort Study. Epilepsy \& Behavior, 59, 92-97. https://doi.org/10.1016/j.yebeh.2016.03.034

[5] Patel, S.I. and Pennell, P.B. (2016) Management of Epilepsy during Pregnancy: An Update. Therapeutic Advances in Neurological Disorders, 9, 118-129. https://doi.org/10.1177/1756285615623934

[6] Siewe, F.J.N., Tatah, G., Tabah, E.N., Ngarka, L., Nfor, L.N., Chokote, S.E., et al. 
(2018) Epidemiology of Onchocerciasis-Associated Epilepsy in the Mbam and Sanaga River Valleys of Cameroon: Impact of More Than 13 Years of Ivermectin. Infectious Diseases of Poverty, 7, 1-11. https://doi.org/10.1186/s40249-018-0497-1

[7] Rajiv, K.R. and Radhakrishnan, A. (2019) Status Epilepticus in Pregnancy-Can We Frame a Uniform Treatment Protocol? Epilepsy \& Behavior, 101, Article ID: 106376. https://doi.org/10.1016/j.yebeh.2019.06.020

[8] Owolabi, L.F., Ibrahim, A., Mohammed, A.D., Owolabi, S.D., et al. (2014) Status Epilepticus in Adults: A Study from Nigeria. International Journal of Epilepsy, 1, 69-74. https://doi.org/10.1016/j.ijep.2014.11.001

[9] Gams, M.D., Endougou, O.C.D., Magnerou, A.M., Kana, A.J., Eko, S.M., Doumbe, J., et al. (2021) Convulsive Status Epilepticus in an Emergency Department in Cameroon. Epilepsy \& Behavior Reports, 16, Article ID: 100440.

https://doi.org/10.1016/j.ebr.2021.100440

[10] Kamyar, M. and Varner, M. (2013) Epilepsy in Pregnancy. Clinical Obstetrics and Gynecology, 56, 330-341. https://doi.org/10.1097/GRF.0b013e31828f2436

[11] (2017) Pregnant Mother in a Coma Wakes Three Months after Delivery. https://www.news-medical.net/news/20171119/Pregnant-mother-in-a-coma-wakesthree-months-after-delivery.aspx

[12] (2019) How Does Someone in a Vegetative State Have a Baby? CNN. https://edition.cnn.com/2019/01/09/health/vegetative-state-pregnancies/index.html

[13] Ministère de la sante publique (2021) Guide de Prise en Charge du Paludisme au Cameroun à l'usage du Personnel de Sante 2021. 